\title{
Validity of the Compassionate Engagement and Action Scales with Family Carers of Older Adults: Confirmatory Factor Analyses
}

\author{
Ms Jenny Murfield ${ }^{1,2}$, Professor Wendy Moyle ${ }^{1,2}$, Professor Analise O'Donovan ${ }^{3}$ and \\ Professor Robert S Ware ${ }^{1,4}$ \\ ${ }^{1}$ Menzies Health Institute Queensland, Griffith University, Brisbane, Australia \\ ${ }^{2}$ School of Nursing and Midwifery, Griffith University, Brisbane, Australia \\ ${ }^{3}$ Griffith Health Group, Griffith University, Brisbane, Australia \\ ${ }^{4}$ School of Medicine, Griffith University, Brisbane, Australia
}

Correspondence should be addressed to: Jenny Murfield, School of Nursing \& Midwifery, Menzies Health Institute Queensland, Griffith University, Health Sciences (N48), 170 Kessels Road, Nathan, Brisbane, Queensland, 4111, Australia. Email: j.murfield@griffith.edu.au 


\section{Validity of the Compassionate Engagement and Action Scales with Family Carers of Older Adults: Confirmatory Factor Analyses}

Objectives: To confirm the factor validity of the Compassionate Engagement and Action Scales, as set out in the original development study, when used with a sample of family carers of older adults.

Design: A series of confirmatory factor analyses were undertaken to test the previously proposed factor solutions of each scale.

Setting: As part of a larger cross-sectional survey, the scales were completed online or via hard copy between July and December 2019.

Participants: An international sample of 171 family carers of adults aged 65 years or older.

Measurements: The Compassionate Engagement and Action Scales are three measures that individually assess Compassion for Self, Compassion to Others, and Compassion from Others. All scales measure two aspects, "engagement" and "actions" (two-factor solution), and Compassion for Self also measures two further dimensions within engagement: "sensitivity to suffering" and "engagement with suffering" (threefactor solution).

Results: Results were largely consistent with the two-factor solutions proposed for the three orientations of compassion, with acceptable fit and good internal reliability. There was some support for the three-factor solution of Compassion for Self; however, despite model fit comparable to the two-factor solution, internal reliability of the delineated 
"engagement" dimensions was low, and there was a weak factor loading for item five that measured distress tolerance.

Conclusions: Use of the Compassionate Engagement and Actions Scales with family carers of older adults is promising. Further research is recommended with larger samples and to explore distress tolerance as a competency within conceptualization and measurement of compassion.

Key words: caring, compassion, factor analysis, family caregiver, psychometrics 


\section{Introduction}

\section{Defining and measuring compassion}

Interest in the scientific study of compassion has rapidly grown over the last two decades (Kirby and Gilbert, 2017); however, there remains no consensus on what compassion is, and various definitions and means of assessment exist (Strauss et al., 2016). In some conceptualizations, compassion is understood as an affective experience and a distinct emotion in itself (Goetz et al., 2010), whereas others define it as a complex multifaceted construct (Strauss et al., 2016). Further still, other conceptualizations assume an evolutionary perspective, including Gilbert (2014), the founder of Compassion-Focused Therapy, who proposes that compassion is an evolved caring motivational system. Drawing on the perspectives of evolutionary psychology, neuroscience, and theories of social mentality and attachment, Gilbert (2014) hypothesizes that compassion is understood as an interactive three-way flow (involving compassion for self, compassion to others, and compassion from others) that evolved to regulate negative affect via the affiliative/soothing system associated with secure attachment and pro-social behaviour. Within this conceptualization, compassion is defined as: "a sensitivity to suffering in self and others, with a commitment to try to prevent or alleviate it" (Gilbert, 2014, p.19). This definition specifically covers the two dimensions of psychological motivational processing, that of attention/engagement (i.e., sensitivity to suffering) and action (i.e., commitment to prevent or alleviate suffering). These two dimensions are referred to as the first and second psychologies of compassion and, within each, specific competencies are outlined as necessary for all three compassion orientations. For the first psychology of compassion - 
"compassionate engagement" - the following six competencies are identified as key attributes required to engage with suffering: care for well-being (i.e., motivation and willingness to care and respond to suffering); sensitivity (i.e., responding to needs when required); distress tolerance (i.e., tolerating difficult emotions without becoming overwhelmed); sympathy (i.e., showing emotional resonance to suffering); empathy (i.e., sharing and attuning to others' emotions); and non-judgement (i.e., accepting and tolerating without condemning). For the second psychology of compassion "compassionate action" - the following six competencies are the transformative skills necessary to help alleviate the suffering: attention (e.g., attention training, mindfulness practice); reasoning (e.g., cognitive re-appraisal); behaviour (e.g., gratitude exercises); feeling (e.g., showing positive or negative emotions, as the situation depends); imagery (e.g., guided imagery exercises); and sensory (e.g. breathing practices, posture).

Gilbert et al. (2017) have operationalized this definition of compassion for measurement via the Compassionate Engagement and Action Scales (CEAS), which includes scales for assessment of compassion for self, compassion to others, and compassion from others. Within all three scales, 10 of the 12 competencies are represented as individual scale items; however, no items represent the sensory or imagery competencies, as these experiences were considered too vague to include as items (Gilbert et al., 2017). Within the original development study (Gilbert et al., 2017), the factor structure of the CEAS was validated in two languages (English and Portuguese) in three samples of university students from the UK, USA, and Portugal. Using exploratory and then confirmatory factor analysis, the CEAS demonstrated acceptable levels of reliability and validity, and findings confirmed that the three 
orientations of compassion could each be measured as either three single factor scales, or as subscales measuring aspects of compassionate "engagement" and "actions".

Although still relatively recent in terms of development, the CEAS are beginning to be used within compassion-focused research. In general population samples from Australia and Singapore (Steindl et al., 2018; Steindl et al., 2019), and in caregivers of people with lung cancer in Taiwan (Hsieh et al., 2019), the CEAS have performed acceptably, with good levels of internal reliability reported (Cronbach's $\alpha$ from 0.79 to 0.95). In contrast, a recent study conducted with female survivors of intimate-partner violence in Colombia reported excluding the CEAS as a planned outcome measure, as participants did not understand multiple items during piloting (Naismith et al., 2020). Such findings highlight the importance of testing a scale's performance within a new population to validate its appropriateness for use. Further, aside from the original development study by Gilbert et al. (2017), no other research is known to have confirmed the factor validity of the CEAS in the English language. It is therefore opportune to explore their psychometric performance within a new, independent sample.

\section{Family carers of older adults}

Extensive research shows that caring for a family member over the age of 65 years can be challenging and stressful (Schulz and Sherwood, 2008; Adelman et al., 2014). In comparison to non-caregiving populations, family carers of older adults are more likely to report both psychological and physical health problems, and higher levels of burden, social isolation, and loneliness (Carers UK, 2019; del-Pino-Casado et al., 2019; 
Whittaker and Gallagher, 2019). Although a range of services are currently available to support carers in their role, the need for better access to professional support, particularly at the level of emotion regulation to aid coping and stress management, has been highlighted (Silva et al., 2013). Psychological interventions that seek to cultivate compassion have shown potential benefits for various populations on a number of outcomes including depression, anxiety, psychological distress, and well-being (Kirby et al., 2017). Early findings from studies with family carers of older adults also show similar promise, with the cultivation of compassion seeming to help modify carer stress through facilitating healthy emotion regulation (Collins et al., 2018; Lloyd et al., 2019;

Hlabangana and Hearn, 2019:). Notably, however, none of these early studies have measured compassion via the CEAS and, thus, their performance validity is currently unknown within the population context of family carers of older adults.

The purpose of this study was to confirm the factor validity of the CEAS when used with a sample of family carers of older adults, with a view to assisting gerontological researchers and clinical practitioners in making informed evidence-based decisions around assessment of compassion. A series of confirmatory factor analyses were undertaken to test the proposed factor solutions of each scale - as set out in the original CEAS development study by Gilbert et al. (2017) - and to assess how well each solution fitted the sample data. Confirmatory factor analysis is a measurement model that tests the relationship between observed manifest variables and unobserved latent variables (i.e., scale items to theoretical constructs). For this reason, confirmatory factor analysis is used to confirm or reject hypothesized factor structures of multi-item, multisubscale instruments (Byrne, 2010). 


\section{Method}

\section{Participants and procedure}

This confirmatory factor analyses of the CEAS was nested within a larger crosssectional survey study that aimed to explore the relationship between compassion (measured via the CEAS) and the mental health and wellbeing of family carers of older adults. Participants completing the CEAS had to meet the larger survey study's inclusion criteria: aged 18 years or older; providing regular, on-going, unpaid care to a family member, friend, or neighbor over the age of 65 years; and able to read and write in English. Participants were recruited through: promotion via advocacy services, and community and volunteer organizations and networks (i.e., e-newsletters, social media posts, discussion at meetings); face-to-face presentations at carer support groups and information sessions; known individuals and carer advocates; social media (i.e., Facebook carer support groups, Twitter); print newspaper advertisements; radio interviews; and word-of-mouth. The survey was available online (hosted through LimeSurvey $\mathrm{GmbH}$, Hamburg, Germany), via hard copy with pre-paid return, or through verbal interview with the lead author.

The survey underwent two rounds of piloting with family carers in June 2019 ( $n=3$ round one; $\mathrm{n}=4$ round two). After round one, minor edits were made to the introductory text and response options for two demographic items. No further changes were made to the survey after round two, and these pilot data $(n=4)$ are included as part of the final sample. The cross-sectional survey was open for a six-month period from July to December 2019. 
Ethical approval was obtained from Griffith University Human Research Ethics Committee (GU ref. no. 2019/481). At the time of completion, all participants were provided with written information about the survey and notified that completion constituted formal consent. Participants were informed that their responses were anonymous and that they were free to stop at any time. The CEAS were placed at the start of the survey after demographic items, and were the first self-report scales that participants completed. The survey was estimated to take 30-minutes and, to minimize burden, participants were encouraged to take breaks during completion. Written reminders to pause completion appeared at two points during the survey, and a saveand-return function was enabled in the online version. Given that the focus of the larger

cross-sectional survey was on the mental health and wellbeing of carers, a link to global online mental health resources was provided, as well as the contact details for individual support organizations around the world.

\section{Measures}

Originally produced in English, the CEAS (Gilbert et al., 2017) is a collection of three, 13-item measures that individually assess compassion in terms of the three flows, namely compassion for self, compassion to others, and compassion from others. Each scale is divided into two sections to measure the first and second psychologies of compassion respectively: compassionate "engagement" and "actions". Within the scale measuring Compassion for Self, there is also the option to analyze two additional dimensions within "engagement," that of "sensitivity to suffering" and "engagement with suffering". Three reverse "filler" items are also included in each scale to mitigate the 
impact of response bias (items 3 and 7 in "engagement"; item 3, referred to as item 11 in this study, in "actions"), and these items are excluded from scoring. All scales use a 10-point Likert scale ( $1=$ "never" to $10=$ "always") to assess the frequency of each item's occurrence. When summated, these form total scale and subscale scores; higher scores represent higher levels of compassion. Within the original development study (Gilbert et al., 2017), the CEAS showed reasonable construct validity (including convergent validity) with other established measures of compassion, and demonstrated good internal reliability (Cronbach's $\alpha$ from 0.67 to 0.94 ). Descriptive statistics and internal consistencies of the CEAS within the current study are provided in Table 1. These are largely comparable to those reported by Gilbert et al. (2017).

A range of demographic and caregiving-related data about the family carer and the older adult care recipient were collected (see Table 2).

[Insert Table 1 about here]

\section{Data analyses}

A sample of $n=189$ family carers of older adults completed the CEAS ( $n=169$ online, including $n=4$ in round two of piloting; $n=20$ printed hard-copy). These data were entered into IBM SPSS Version 26 (IBM Corp, Armonk, NY) for analysis. The online survey automatically produced an SPSS data file, thereby eliminating the need for data entry checks; however, accuracy of all manually entered data from hard-copy surveys was rechecked against raw data. Basic descriptive statistics were produced for all variables to explore missing values and to check the initial spread of responses. On inspection, $n=13$ cases did not meet all participant inclusion criteria, and a further $n=5$ 
cases had excessive amounts of missing data (defined as $>20 \%$ data missing across the three CEAS, with amounts ranging from $23.3 \%$ to $60 \%$ ). These cases were removed. Within the final sample of $n=171$, missing values analyses showed small amounts of missing data (across CEAS $=0.9 \%$; compassion for self $=0.6 \%$; compassion to others $=0.4 \%$; compassion from others $=1.7 \%$ ). These data appeared to be Missing Completely at Random (Little's MCAR test: across CEAS $p=.304$; compassion for self $p=.330$; compassion to others $p=.406$; compassion from others $p=.353$ ), and could be approximated with a normal distribution (i.e., no instances of observed univariate skewness $>2.0$ and kurtosis $>7.0$ (Curran et al., 1996)). Based on this data profile, the expectation-maximization algorithm was deemed appropriate to impute missing values on the CEAS (Bennett, 2001). No imputation was applied to demographic variables. CEAS totals and subscale totals were calculated, and descriptive statistics and internal consistency estimates (Cronbach's $\alpha$ ) were produced. Bivariate correlations were also undertaken to assess, within each scale, the level of association between total and subscales.

To confirm the factor validity of the three CEAS, a series of confirmatory factor analyses were conducted in the manner described in the original development study (i.e., higher-order factor analysis) using the structural equation modelling program, IBM SPSS AMOS Version 26.0 (IBM Corp, Armonk, NY). Model parameters were estimated using maximum likelihood methods due to the normal distribution of data (Kline, 2015). In determining the extent to which each proposed model fitted the sample data, parameter estimates were first inspected for feasibility and acceptability (loadings of $> \pm$ .40 or greater (Yang, 2010)), appropriateness of standard errors, and statistical 
significance $(p<0.05)$. Goodness-of-fit statistics were inspected, as were standardized residual covariances for signs of potential model misspecification. Goodness-of-fit indices were chosen based on their stability and performance when used with sample sizes of less than $n=200$ (Byrne, 2010; Brown, 2015). Further, as recommended when conducting confirmatory factor analyses with smaller samples, multiple fit indices were considered in relation to model fit under absolute, parsimonious, and comparative conditions, and indices cut-offs were interpreted using close-to rather than definitive thresholds (Hu and Bentler, 1999; Brown, 2015). With this in mind, the following five goodness-of-fit indices, and their respective criteria for good levels of model fit, were applied (Byrne, 2010). For statistical model fit, the Chi-square statistic $\left(X^{2}\right)$ was used, with non-significant values of $p>0.05$ indicating good levels of fit (Byrne, 2010). However, given that this statistic is known to be problematic in fitting real-world data, with significant results often found even in well-fitting models (Byrne, 2010), a number of pragmatic indices were also used. These indices and their desired values are as follows: Normed Chi-Square statistic ( $\left.x^{2} / \mathrm{df}\right)$ of less than $<3$ but no greater than $>5$ (Wheaton et al., 1977); Standardized Root Mean Square Residual (SRMR) of <.08 (Hu and Bentler, 1999); Comparative Fit Index (CFI) of close-to or between .90 and .95 (Brown, 2015); and Parsimony Comparative Fit Index (PCFI) expected around $>.50$ when $\mathrm{CFI}$ are within the specified range of close to or between .90 and .95 (Byrne, 2010). It should be noted that, given their known sensitivity to smaller samples and resulting tendency to over-reject true-population models, the often reported TuckerLewis Index (TLI), Root Mean Squared Error of Approximation (RMSEA), and Goodness-of-Fit Index (GFI) were not used to assess model fit for any of the conducted 
confirmatory factor analyses (Hu and Bentler, 1999). Finally, evidence of model misspecification was explored via inspection of each model's standardized residual covariances (which are comparable to Z-scores and represent the estimated number of standard deviations from zero residuals if perfect model fit was achieved). A cut-off of $>2.58$ was used to indicate statistically significant discrepancy suggestive of necessary post-hoc model modification (Joreskog and Sorbom, 1993).

\section{Results}

\section{Sample characteristics}

The demographic and caregiving profile of participants is shown in Table 2. Overall, participants completing the CEAS had a mean age of $59.4( \pm 11.5)$ years, were female (87.7\%), lived in Australia (76.0\%), had post-secondary qualifications (either certificate/diploma $42.9 \%$ or university degree or higher $43.5 \%$ ), and were either retired $(39.2 \%)$ or in some from of employment $(34.5 \%)$. Most were primary carers $(86.0 \%)$, who lived full-time (64.9\%) with either a parent $(54.4 \%)$ or partner $(31.0 \%)$, and had been in the caregiving role for more than three years (68.1\%). The majority of participants provided care to a female $(58.5 \%)$, with an average age of $80.9( \pm 8.8)$ years, and who were most likely to have Alzheimer' disease or dementia (62.6\%), a musculoskeletal disorder (33.9\%), and/or cardiovascular disease (19.3\%).

[Insert Table 2 about here]

\section{Confirmatory factor analyses}

CEAS: COMPASSION FOR SELF 
For the CEAS Compassion for Self scale, two models were tested for fit, as consistent with the original theoretical and empirical evidence presented by Gilbert et al. (2017). In the first model, two first-order latent factors ("engagement" and "actions") were loaded onto the second higher-order latent factor of Compassion for Self. This proposed construct of Compassion for Self as dimensions of "engagement" and "actions" was supported, with significant positive correlations found between "engagement" and "actions" ( $r=.605, p<0.001)$, as well as between Compassion for Self and "engagement" $(r=.903, p<0.001)$ and "actions" $(r=.888, p<0.001)$. In general, goodness-of-fit indices showed reasonable fit between the model and the observed data (see Table 3). Although statistical fit was not achieved $\left(x^{2}=p<0.001\right)$ - a result not unexpected given the reported problematic nature of this statistic in fitting real-world data (Byrne, 2010) pragmatic indices were all within the applied cut-off values indicative of reasonable model fit. Parameter estimates (see Figure 1a) were all statistically significant at $p<0.05$, although standardized loadings for items 4 and 5 were notably weak (.19 and .25 respectively), and there was no sign of model misspecification when looking at the standardized residual covariances (values all <2.58). As such, no post-hoc modifications were undertaken, and a two-factor solution was supported.

In the second model, a three-factor solution was tested seeing the "engagement" factor analyzed in terms of its two proposed dimensions ("sensitivity to suffering" and "engagement with suffering"). Specifically, the two first-order latent factors ("sensitivity to suffering" and "engagement with suffering") were loaded onto the second-order latent factor of "engagement" that, along with the factor of "actions", was then loaded onto the third-order factor of Compassion for Self. Appropriateness of this construct structure 
was confirmed with significant correlations between all subscales, and between all subscales and the total scale score: "engagement" and "sensitivity to suffering" ( $r=.726$, $p<0.001)$; "engagement" and "engagement with suffering" $(r=.909, p<0.001)$; "sensitivity to suffering" and "engagement with suffering" ( $r=.372, p<0.001)$; "sensitivity to suffering" and "actions" ( $r=.182, p<0.05)$; "engagement with suffering" and "actions" $(r=.705$, $p<0.001)$; and "sensitivity to suffering" and Compassion for Self $(r=.517, p<0.001)$; "engagement with suffering" and Compassion for Self $(r=.905, p<0.001)$. As shown in Table 3, the goodness-of-fit indices indicated a reasonable fit for the three-factor model and the observed data, particularly from a pragmatic perspective, and were comparable to the two-factor model. Parameter estimates (see Figure 1b) were all statistically significant (all $p<0.05)$, although item 5 still indicated weak loading (.28), and no issues were apparent with the standardized residual covariances (all <2.58). Given these data, post-hoc modifications were not deemed necessary, and this three-factor solution was supported.

[Insert Table 3 about here] \& [Insert Figure 1 about here]

\section{CEAS: COMPASSION TO OTHERS}

For the CEAS Compassion to Others scale, the two-factor solution proposed by Gilbert et al. (2017) was tested, seeing the two first-order latent factors ("engagement" and "actions") loaded onto the second higher-order latent factor of Compassion to Others. This proposed construct of Compassion to Others as dimensions of "engagement" and "actions" was supported, with significant positive correlations found between "engagement" and "actions" $(r=.793, p<0.001)$, as well as between Compassion to 
Others and "engagement" $(r=.965, p<0.001)$ and "actions" $(r=.925, p<0.001)$. As presented in Table 3, the pragmatic goodness-of-fit indices showed generally reasonable fit between the model and the observed data, although these tended towards the marginal close-to ranges rather than definitive indices cut-offs, and statistical fit was not achieved. The parameter estimates shown in Figure $2 \mathrm{c}$ were all statistically significant at $p<0.05$, and above the desired cut-off of .40 ; however, item 5 again had the weakest loading of all items (.52). Analysis of the standardized residual covariances suggested no concern for model misspecification (values all <2.58), deeming post-hoc modifications unnecessary, and the model supportive of a two-factor solution.

[Insert Figure 2 about here]

\section{CEAS: COMPASSION FROM OTHERS}

For the CEAS Compassion from Others scale, the two-factor solution originally proposed by Gilbert et al. (2017) was tested in which two first-order latent factors ("engagement" and "actions") were loaded onto a second higher-order latent factor of Compassion from Others. Support for the proposed construct of Compassion from Others as dimensions of "engagement" and "actions" was evidenced in significant positive correlations between "engagement" and "actions" ( $r=.883, p<0.001)$, as well as between Compassion from Others and "engagement" $(r=.978, p<0.001)$ and "actions" $(r=.961, p<0.001)$. Goodness-of-fit indices indicated acceptable-to-good levels of model fit to the observed data (see Table 3), with pragmatic indices found within the desired cut-off ranges. Parameter estimates were also all significant (see Figure 2d), although 
item 5 again had the weakest loading (.64), and standardized residual covariances were all within the desired range of $<2.58$. These data were considered supportive of a twofactor solution, and no post-hoc modifications were undertaken for model misspecification.

\section{Discussion}

This study used confirmatory factor analyses to test the factor structures of the CEAS, as proposed by Gilbert et al. (2017), within an international sample of $n=171$ family carers of adults aged 65 years or older with a broad range of health conditions. To the best of our knowledge, this is the first study to use the CEAS within this population, and is the first independent study conducted, post-scale development, to examine the proposed factor structures.

Overall, findings from the confirmatory factor analyses lend preliminary support for the CEAS as measures of compassion for self, compassion to others, and compassion from others, with results largely consistent with the factor solutions proposed by Gilbert et al. (2017). Within all three orientations, there was support for the measurement of compassion as an overarching construct made-up of the dimensions, "engagement" and "actions". Specifically, we found positive and significant correlations between total and subscales within each scale, and all the two-factor solutions that we tested showed pragmatically acceptable levels of fit, significant factor loadings, and no evidence of model misspecification. There were also good-to-excellent levels of internal reliability for each scale when measuring compassion using total scores, and when 
using the two subscales of "engagement" and "actions" (Cronbach's $\alpha$ ranging from 0.70 to 0.96$)$.

For the scale of Compassion for Self, there was also some support for the threefactor solution proposed by Gilbert et al. (2017), whereby "engagement" was delineated further into two dimensions: "sensitivity to suffering" and "engagement with suffering". This three-factor solution fitted the data at a similarly acceptable level to the two-factor solution. We, therefore, recommend that both the two- and three- factor solutions can appropriately measure compassion for self and suggest that the choice of the solution selected should depend on alignment with the aims and purpose of assessment. Nevertheless, when using this three-factor solution, the internal reliabilities of these two additional dimensions were on the low side of acceptability (Cronbach's $\alpha$ "sensitivity to suffering" $=0.57$; "engagement with suffering" $=0.67$ ). In addition, there was also a particularly weak factor loading for item five ("I tolerate the various feelings that are a part of my distress": standardized parameter estimate $=.28$ ); and this was also the weakest loading item across all scales (standardized parameter estimates for Compassion to Others $=.52$, and Compassion from Others $=.64)$. Issues with items measuring aspects of tolerating distress have been reported in previous research exploring compassion, to both self and others (Gu et al., 2017), whereby a similar pattern to our results was reported: weak factor loadings, despite good model fit indices and significant factor loadings. Gu et al. (2017) recommended further work to explore whether their findings were representative of item-level issues, or whether they represented wider factor-level issues around including tolerating within conceptualizations of compassion. In this subsequent research to develop a new 
measure of compassion (Gu et al., 2019) - the Sussex-Oxford Compassion Scales - no issues were found for newly generated items representing the factor "tolerating" (which suggested that previous findings were item-level and not factor-level issues), and the factor was maintained in their five-dimension understanding of compassion.

Within the context of our study findings, the CEAS represent distress tolerance by a single item - item five. The weak factor loading may be indicative of an issue with this item specifically (although the acceptable pragmatic model-of-fit statistics, significant factor loadings, and absence of model misspecification make this interpretation less clear, and are also the reasons why we did not seek to exclude this item in post-hoc model modifications). Additional exploratory work would be helpful to determine whether issues such as inadequate wording of the item contributed to the low factor loading. Yet, it is certainly notable that we found a similar issue to another study and which used a different measure of compassion - around the aspect of distress tolerance. Further work is warranted to explore the specific association between tolerating uncomfortable feelings and conceptualization of the three orientations of compassion.

\section{Limitations}

In interpreting these study findings, a number of limitations should to be kept in mind. First, a sample of less than 200 is considered small for conducting confirmatory factor analyses, and a sample of 210 would have been desirable for each model that we tested (based on $n=10$ for each of the 21 distinct parameters to be estimated (Byrne, 2010)). Nevertheless, to ensure that we did not produce over-specified models based 
on the idiosyncrasies of a smaller-than-desirable sample, a pragmatic approach was used to guide the analysis and interpretation (Byrne, 2010; Brown, 2015). Specifically, this included the decision to use sample-sensitive goodness of fit tests that covered multiple measures of fit in terms of absolute, parsimonious and comparative conditions, and to use close-to rather than absolute indices cut-offs (Hu and Bentler, 1999; Byrne, 2010; Brown, 2015). Second, there is evidence that surveys completed in different response formats (i.e., online or paper-and pencil) can produce different results (Zhang et al., 2017). Within this study, the vast majority of survey responses were completed online, and a minority were completed by hand. Findings may have been different, therefore, if other response methods were used to complete the survey, and particularly for methods other than online completion.

\section{Implications}

In terms of future research, additional studies are needed to build on these promising findings and confirm the results we found in this study when using a larger sample. In doing so, researchers should also consider the psychometric performance of the CEAS more broadly, exploring the convergent validity of the scale when compared with other scales that measure compassion. In addition, studies should consider exploring the unique effect of different participant factors on the scales' performance. Within transactional models of caregiver stress, background and contextual factors (e.g., characteristics of the carer), as well as type of stressor (e.g., care needs of the care recipient) and, more recently, cultural and ethnicity-based values, have been shown to influence carers' stress (Lazarus and Folkman, 1984; Pearlin et al., 1990; Knight and 
Sayegh, 2010). As such, future studies would benefit from either being more targeted in their recruitment of participants with specific demographic or clinical profiles, or by seeking to recruit large enough samples to accommodate more nuanced analyses. Finally, greater exploration of item five is needed within the CEAS, which measures the aspect of distress tolerance. Studies using confirmatory factor analyses with larger samples may, if indicated as appropriate by their sample data, consider excluding item five in post-hoc model modifications to determine if model fit is improved. Further, given that issues around the measurement of tolerating has been found in other compassion scale studies (Gu et al., 2017), we also recommend additional qualitative work should be undertaken to delve further into understanding how tolerating uncomfortable feelings fits within conceptualizations of the three orientations of compassion.

The cultivation of compassion is a rapidly growing area of scientific inquiry in the field of mental health and adaptive emotion regulation, and it is newly emerging in terms of informal carers of older adults (Murfield et al., 2019). As such, it is important that gerontological researchers and clinical practitioners have evidence-based data that they can use to help their decisions around if, and how, they might assess compassion within family carers of older adults. This study reports the first known data on the factor validity of the CEAS and provides preliminary evidence to support their use as two-factor solutions for all scales, as well as the three-factor solutions for the scale Compassion for Self. To date, no other suite of scales is available to measure all three orientations of compassion together, making these early findings particularly helpful to those interested in exploring compassion as it relates to the three flows. 


\section{Conclusion}

This study provides promising support for the use of the CEAS with family carers of older adults. Further research is recommended to explore the psychometric performance of the scales in larger samples, as well as further exploration of distress tolerance within conceptualizations and measurement of compassion.

\section{Conflict of interest}

None.

\section{Description of authors' roles}

J. Murfield conceived and designed the study, undertook all data collection and statistical analysis, and wrote, revised, and finalized the paper. W. Moyle and A. O'Donovan supervised the study, providing advice in aspects of study design and data collection, and conceptually commented on and revised the paper. R. Ware provided statistical advice on data analysis, and conceptually commented on and revised the paper.

\section{Acknowledgments}

This work was supported by a Griffith University Postgraduate Scholarship. Thanks are expressed to the organisations, services, and online support groups who assisted with promotion of the survey, and to the family carers who took the time to complete the survey. 


\section{References}

Adelman, R. D., Tmanova, L. L., Delgado, D., Dion, S. and Lachs, M. S. (2014).

Caregiver burden: A clinical review. Journal of the American Medical Association, 311, 1052-1060. doi: 10.1001/jama.2014.304.

Bennett, D. A. (2001). How can I deal with missing data in my study? Australian and New Zealand Journal of Public Health, 25, 464-469. doi: 10.1111/j.1467842X.2001.tb00294.x.

Brown, T. A. (2015). Confirmatory Factor Analysis for Applied Research. New York, USA: Guilford Publications, Inc.

Byrne, B. (2010). Structural Equation Modeling with AMOS: Basic Concepts, Applications, and Programming. New York, USA: Routledge.

Carers UK 2019. State of caring: A snapshot of unpaid care in the UK. Retrieved from http://www.carersuk.org/stateofcaring-report; last accessed 13 March 2020.

Collins, R. N., Gilligan, L. J. and Poz, R. (2018). The evaluation of a compassionfocused therapy group for couples experiencing a dementia diagnosis. Clinical Gerontologist, 41, 474-486. doi: 10.1080/07317115.2017.1397830.

Curran, P. J., West, S. G. and Finch, J. F. (1996). The robustness of test statistics to nonnormality and specification error in confirmatory factor analysis. Psychological Methods, 1, 16-29. doi: 10.1037/1082-989X.1.1.16.

del-Pino-Casado, R., Rodríguez Cardosa, M., López-Martínez, C. and Orgeta, V. (2019). The association between subjective caregiver burden and depressive symptoms 
in carers of older relatives: A systematic review and meta-analysis. PLOS ONE, 14, e0217648. doi: 10.1371/journal.pone.0217648.

Gilbert, P. (2014). The origins and nature of compassion focused therapy. British Journal of Clinical Psychology, 53, 6-41. doi: 10.1111/bjc.12043.

Gilbert, P. et al. (2017). The development of compassionate engagement and action scales for self and others. Journal of Compassionate Health Care, 4, 4. doi:

10.1186/s40639-017-0033-3.

Goetz, J. L., Keltner, D. and Simon-Thomas, E. (2010). Compassion: An evolutionary analysis and empirical review. Psychological Bulletin, 136, 351-374. doi: 10.1037/a0018807.

Gu, J., Baer, R., Cavanagh, K., Kuyken, W. and Strauss, C. (2019). Development and psychometric properties of the Sussex-Oxford Compassion Scales (SOCS). Assessment, 27, 3-20. doi: 10.1177/1073191119860911.

Gu, J., Cavanagh, K., Baer, R. and Strauss, C. (2017). An empirical examination of the factor structure of compassion. PLOS ONE, 12, e0172471. doi: 10.1371/journal.pone.0172471.

Hlabangana, V. and Hearn, J. H. (2019:). Depression in partner caregivers of people with neurological conditions; associations with self-compassion and quality of life. Journal of Mental Health, 1-6. doi: 10.1080/09638237.2019.1630724. Hsieh, C.-C., Yu, C.-J., Chen, H.-J., Chen, Y.-W., Chang, N.-T. and Hsiao, F.-H. (2019). Dispositional mindfulness, self-compassion, and compassion from others as moderators between stress and depression in caregivers of patients with lung cancer. Psycho-Oncology, 28, 1498-1505. doi: 10.1002/pon.5106. 
Hu, L. and Bentler, P. M. (1999). Cutoff criteria for fit indexes in covariance structure analysis: Conventional criteria versus new alternatives. Structural Equation Modeling: $A$ Multidisciplinary Journal, 6, 1-55. doi: 10.1080/10705519909540118.

Joreskog, K. and Sorbom, D. (1993). LISREL 8: Structural Equation Modeling with the SIMPLIS Command Language Chicago, USA: Scientific Software International, Inc.

Kirby, J. N. and Gilbert, P. (2017). The emergence of the compassion focused therapies. In P. Gilbert (Ed.) Compassion: Concepts, Research and Applications (pp. 258-285) London, UK: Routledge.

Kirby, J. N., Tellegen, C. L. and Steindl, S. R. (2017). A meta-analysis of compassionbased interventions: Current state of knowledge and future directions. Behavior Therapy, 48, 778-792. doi: 10.1016/j.beth.2017.06.003.

Kline, R. B. (2015). Principles and Practice of Structural Equation Modeling. New York, USA: Guilford Publications, Inc.

Knight, B. G. and Sayegh, P. (2010). Cultural values and caregiving: The updated sociocultural stress and coping model. The Journals of Gerontology. Series B, Psychological Sciences and Social Sciences, 65b, 5-13. doi: 10.1093/geronb/gbp096.

Lazarus, R. S. and Folkman, S. (1984). Stress, Appraisal, and Coping. New York, USA: Springer Publishing.

Lloyd, J., Muers, J., Patterson, T. G. and Marczak, M. (2019). Self-compassion, coping strategies, and caregiver burden in caregivers of people with dementia. Clinical Gerontologist, 42, 47-59. doi: 10.1080/07317115.2018.1461162. 
Murfield, J., Moyle, W. and O'Donovan, A. (2019). Mindfulness- and compassionbased interventions for family carers of older adults: A scoping review. International Journal of Nursing Studies, 103495. doi: 10.1016/j.jnurstu.2019.103495.

Naismith, I., Ripoll, K. and Pardo, V. M. (2020). Group compassion-based therapy for female survivors of intimate-partner violence and gender-based violence: A pilot study. Journal of Family Violence. doi: 10.1007/s10896-019-00127-2.

Pearlin, L. I., Mullan, J. T., Semple, S. J. and Skaff, M. M. (1990). Caregiving and the stress process: An overview of concepts and their measures. The Gerontologist, 30, 583-594. doi: 10.1093/geront/30.5.583.

Schulz, R. and Sherwood, P. R. (2008). Physical and mental health effects of family caregiving. American Journal of Nursing, 108, 23-27. doi:

10.1097/01.NAJ.0000336406.45248.4c.

Silva, A. L., Teixeira, H. J., Teixeira, M. J. and Freitas, S. (2013). The needs of informal caregivers of elderly people living at home: An integrative review. Scandinavian Journal of Caring Sciences, 27, 792-803. doi: 10.1111/scs.12019.

Steindl, S. R., Matos, M. and Creed, A. K. (2018). Early shame and safeness memories, and later depressive symptoms and safe affect: The mediating role of selfcompassion. Current Psychology. doi: 10.1007/s12144-018-9990-8.

Steindl, S. R., Yiu, R. X. Q., Baumann, T. and Matos, M. (2019). Comparing compassion across cultures: Similarities and differences among Australians and Singaporeans. Australian Psychologist. doi: 10.1111/ap.12433. 
Strauss, C. et al. (2016). What is compassion and how can we measure it? A review of definitions and measures. Clinical Psychology Review, 47, 15-27. doi:

10.1016/j.cpr.2016.05.004.

Wheaton, B., Muthen, B., Alwin, D. F. and Summers, G. F. (1977). Assessing reliability and stability in panel models. Sociological Methodology, 8, 84-136. doi: $10.2307 / 270754$.

Whittaker, A. C. and Gallagher, S. (2019). Caregiving alters immunity and stress hormones: A review of recent research. Current Opinion in Behavioral Sciences, 28, 9397. doi: 10.1016/j.cobeha.2019.02.002.

Yang, H. (2010). Factor loadings. In N. J. Salkind (Ed.) Encyclopedia of Research Design Thousand Oaks, California, USA: SAGE Publications, Inc.

Zhang, X., Kuchinke, L., Woud, M. L., Velten, J. and Margraf, J. (2017). Survey method matters: Online/offline questionnaires and face-to-face or telephone interviews differ. Computers in Human Behavior, 71, 172-180. doi: 10.1016/j.chb.2017.02.006. 


\section{Figure and table legends}

Figure 1. Standardized parameter estimates from the confirmatory factor analyses of the Compassionate Engagement and Actions Scales with (A) Compassion for Self twofactor solution, and (B) Compassion for Self three-factor solution. Rectangles represent the 10 items comprising the scale, ovals represent the latent factors, and circles represent error and residual variances.

Figure 2. Standardized parameter estimates from the confirmatory factor analyses of the Compassionate Engagement and Actions Scales with (C) Compassion to Others two-factor solution, and (D) Compassion from Others two-factor solution. Rectangles represent the 10 items comprising the scale, ovals represent the latent factors, and circles represent error and residual variances.

Table 1. Descriptive statistics and internal consistencies of the three Compassionate Engagement and Action Scales

Note. CEAS = Compassionate Engagement and Action Scales. Within all scales, items were rated using a 10-point Likert response scale, whereby $1=$ Never and 10= Always. Higher scores represent higher levels of compassion. 
Table 2. Demographic and caregiving profile of participants

Note. Continuous variables are reported as mean and standard deviation ( $\pm S D$ ). Categorical variables are reported as $n$ (\%) Frequencies and proportions may not add up to $n=171$ and $100 \%$ due to missing data, rounding, or multiple response options.

Table 3. Models of fit summaries from the confirmatory factor analyses of the three Compassionate Engagement and Action Scales

Note. CEAS = Compassionate Engagement and Action Scales; $\mathrm{X} 2$ = Chi-square statistic, with desired values of $p>0.05$ (non sig.); $d f=$ degrees of freedom; $p=p$-value; $x 2 / d f=$ Normed Chi-Square statistic, with desired values of $<3$ but no $>5$; SRMR $=$ Standardized Root Mean Square Residual, with desired values of <.08; CFI = Comparative Fit Index, with desired values of close-to or between .90 and .95; PCFI

$=$ Parsimony Comparative Fit Index, with values expected around $>.50$ when CFI are within the specified range of close to or between .90 and .95 . 
A.

B.

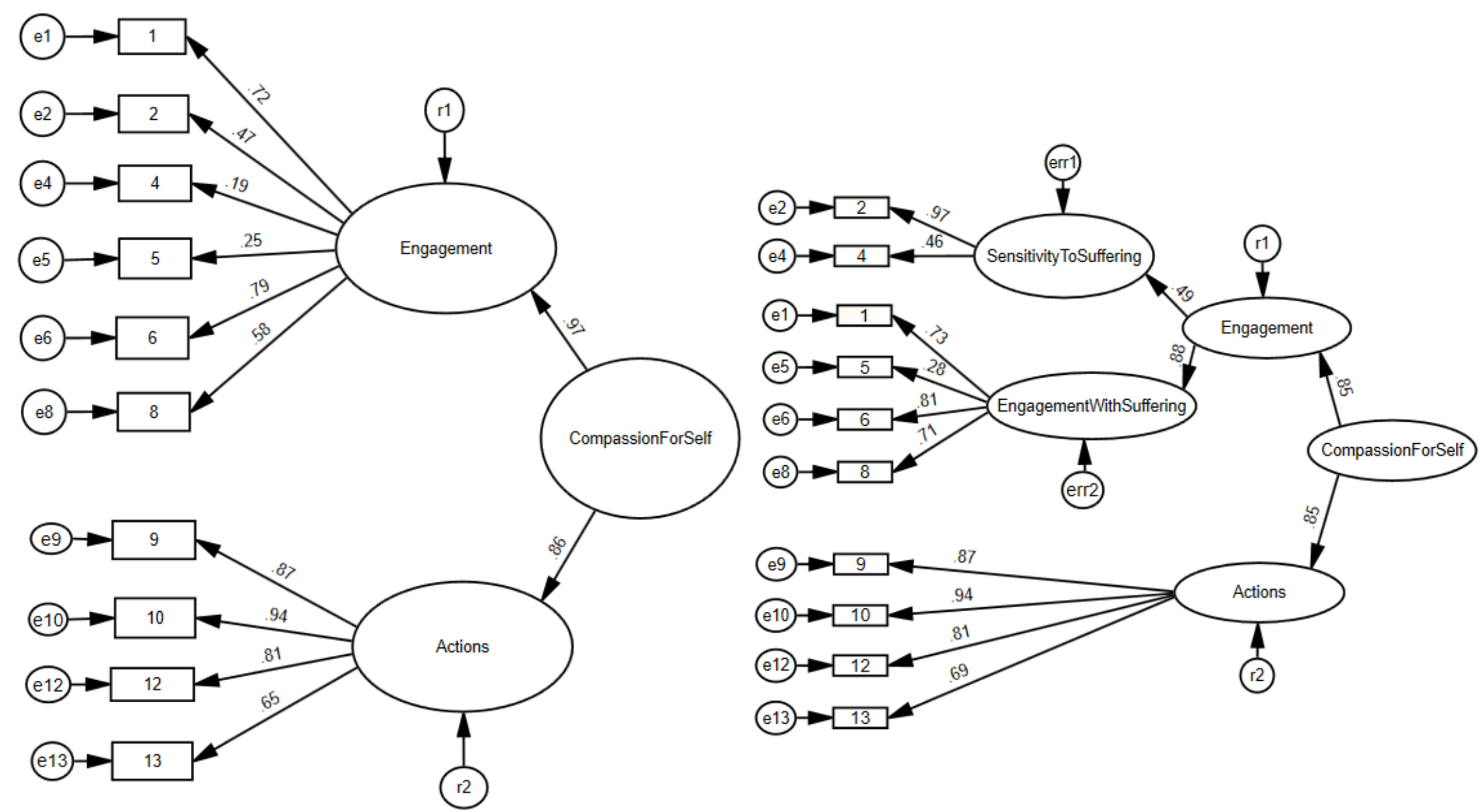

Figure 1. Standardized parameter estimates from the confirmatory factor analyses of the Compassionate Engagement and Action Scales with (A) Compassion for Self two-factor solution, and (B) Compassion for Self three-factor solution. Rectangles represent the 10 items comprising the scale, ovals represent the latent factors, and circles represent error and residual variances. 
C.

D.
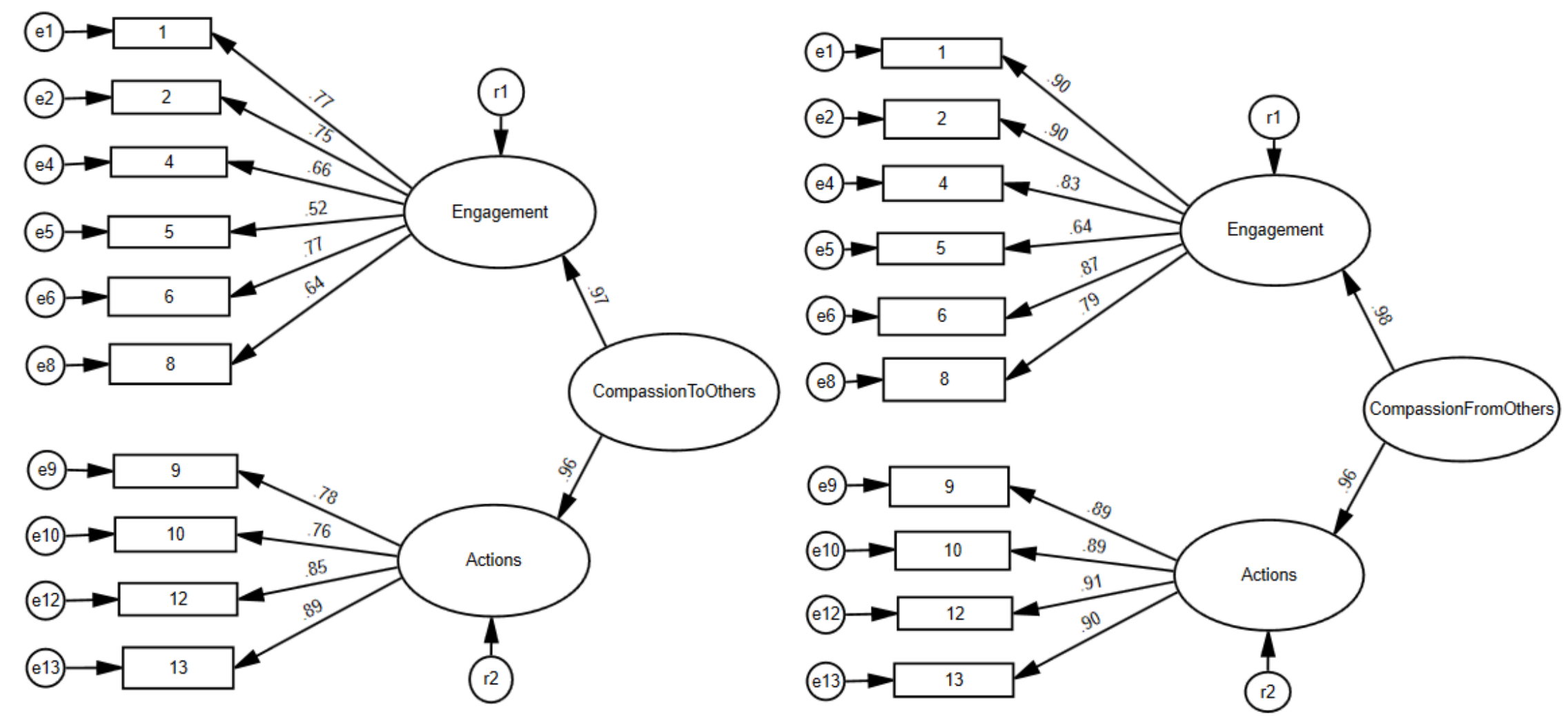

Figure 2. Standardized parameter estimates from the confirmatory factor analyses of the Compassionate Engagement and Actions Scales with (C) Compassion to Others two-factor solution, and (D) Compassion from Others two-factor solution. Rectangles represent the 10 items comprising the scale, ovals represent the latent factors, and circles represent error and residual variances. 
Table 1. Descriptive statistics and internal consistencies of the three Compassionate

Engagement and Action Scales

\begin{tabular}{lll}
\hline Scale/ subscale & \multicolumn{2}{c}{ Family carers of older adults } \\
& Mean $( \pm$ SD $)$ & $\begin{array}{c}\mathrm{n}=171 \\
\text { Cronbach's } \alpha\end{array}$ \\
& & \\
CEAS: Compassion for self & $60.42(15.70)$ & .85 \\
$\quad$ Scale total & $36.70(9.07)$ & .70 \\
Engagement subscale & $13.29(4.08)$ & .57 \\
$\quad$ Sensitivity to suffering & $23.41(6.72)$ & .67 \\
$\quad$ Engagement with suffering & $23.71(8.45)$ & .89 \\
Actions subscale & & \\
CEAS: Compassion to others & $78.02(13.71)$ & .91 \\
$\quad$ Scale total & $46.05(8.56)$ & .84 \\
Engagement subscale & $31.97(5.89)$ & .89 \\
Actions subscale & & \\
CEAS: Compassion from others & $52.29(21.61)$ & .96 \\
$\quad$ Scale total & $31.07(12.69)$ & .93 \\
Engagement subscale & $21.22(9.56)$ & .94 \\
$\quad$ Actions subscale & .
\end{tabular}

Note. CEAS = Compassionate Engagement and Action Scales. Within all scales, items were rated using a 10-point Likert response scale, whereby $1=$ Never and $10=$ Always. Higher scores represent higher levels of compassion. 
Table 2. Demographic and caregiving profile of participants

\begin{tabular}{|c|c|}
\hline Characteristic & $\mathrm{n}=171$ \\
\hline Age (years) & $59.4( \pm 11.5)$ \\
\hline \multicolumn{2}{|l|}{ Sex } \\
\hline Female & $150(87.7)$ \\
\hline Male & $20(11.7)$ \\
\hline \multicolumn{2}{|l|}{ Highest level of education } \\
\hline University degree or higher & $74(43.5)$ \\
\hline Post-secondary certificate or diploma & 73 (42.9) \\
\hline Secondary & $22(12.9)$ \\
\hline Primary & $1(0.6)$ \\
\hline \multicolumn{2}{|l|}{ Employment status } \\
\hline Employed & $59(34.5)$ \\
\hline Unemployed (not seeking work) & $35(20.5)$ \\
\hline Unemployed (seeking work) & $8(4.7)$ \\
\hline Retired & $67(39.2)$ \\
\hline Student & $5(2.9)$ \\
\hline \multicolumn{2}{|l|}{ Country of residence } \\
\hline Australia & $130(76.0)$ \\
\hline United States of America & $22(12.9)$ \\
\hline United Kingdom & $13(7.6)$ \\
\hline Canada & $2(1.2)$ \\
\hline China & $1(0.6)$ \\
\hline Kenya & $1(0.6)$ \\
\hline Sweden & $1(0.6)$ \\
\hline \multicolumn{2}{|l|}{ Relationship to older adult } \\
\hline Offspring & $93(54.4)$ \\
\hline Partner & $53(31.0)$ \\
\hline Relative & $15(8.8)$ \\
\hline Sibling & $5(2.9)$ \\
\hline Friend or neighbor & $3(1.8)$ \\
\hline \multicolumn{2}{|l|}{ Type of carer } \\
\hline Primary carer & $147(86.0)$ \\
\hline Secondary carer & $22(12.9)$ \\
\hline \multicolumn{2}{|l|}{ Living full-time with older adult } \\
\hline Yes & $111(64.9)$ \\
\hline No & $59(34.5)$ \\
\hline Length of time caring (years) & $6.17( \pm 5.65)$ \\
\hline Age of older adult (years) & $80.9( \pm 8.8)$ \\
\hline \multicolumn{2}{|l|}{ Sex of older adult } \\
\hline Female & $100(58.5)$ \\
\hline Male & $65(38.0)$ \\
\hline \multicolumn{2}{|l|}{ Chronic condition/s of older adult } \\
\hline Alzheimer's disease or dementia & $107(62.6)$ \\
\hline Musculoskeletal disorders & $58(33.9)$ \\
\hline
\end{tabular}


Cardiovascular disease

Parkinson's disease

Cancer

Lung and respiratory conditions

Mental health conditions

Acquired brain injury

Ear and hearing conditions

Eye and vision conditions

Kidney and bladder conditions

Inflammatory bowel disease

Intellectual disability

Multiple sclerosis
$33(19.3)$

$15(8.8)$

$14(8.2)$

7 (4.1)

7 (4.1)

4 (2.3)

4 (2.3)

4 (2.3)

$3(1.8)$

3 (1.8)

2 (1.2)

2 (1.2)

Note. Continuous variables are reported as mean and standard deviation ( \pm SD). Categorical variables are reported as $n(\%)$. Frequencies and proportions may not add up to 171 and $100 \%$ due to missing data, rounding, or multiple response options. 
Table 3. Models of fit summaries from the confirmatory factor analyses of the three Compassionate Engagement and Action Scales

\begin{tabular}{|c|c|c|c|c|c|c|c|}
\hline \multirow[t]{2}{*}{ Model } & \multicolumn{5}{|c|}{ Absolute } & \multirow{2}{*}{$\begin{array}{c}\text { Compara } \\
\text { tive } \\
\text { CFI }\end{array}$} & \multirow{2}{*}{$\begin{array}{l}\text { Parsimo } \\
\text { nious } \\
\text { PCFI }\end{array}$} \\
\hline & $x^{2}$ & $d f$ & $p$ & $\begin{array}{l}x^{2} / \\
d f\end{array}$ & $\begin{array}{l}\text { SR } \\
\text { MR }\end{array}$ & & \\
\hline $\begin{array}{l}\text { CEAS: Compassion for self (2- } \\
\text { factor solution) }\end{array}$ & $\begin{array}{l}107 . \\
82\end{array}$ & 34 & $\begin{array}{l}0.0 \\
01\end{array}$ & $\begin{array}{c}3.1 \\
7\end{array}$ & .07 & .90 & .68 \\
\hline $\begin{array}{l}\text { CEAS: Compassion for self (3- } \\
\text { factor solution) }\end{array}$ & $\begin{array}{c}122 . \\
72\end{array}$ & 35 & $\begin{array}{l}0.0 \\
01\end{array}$ & $\begin{array}{c}3.5 \\
1\end{array}$ & .08 & .89 & .69 \\
\hline $\begin{array}{l}\text { CEAS: Compassion to others (2- } \\
\text { factor solution) }\end{array}$ & $\begin{array}{c}153 . \\
78\end{array}$ & 34 & $\begin{array}{l}0.0 \\
01\end{array}$ & $\begin{array}{c}4.5 \\
2\end{array}$ & .06 & .89 & .67 \\
\hline $\begin{array}{l}\text { CEAS: Compassion from others (2- } \\
\text { factor solution) }\end{array}$ & $\begin{array}{c}106 . \\
18\end{array}$ & 34 & $\begin{array}{l}0.0 \\
01\end{array}$ & $\begin{array}{c}3.1 \\
2\end{array}$ & .03 & .96 & .72 \\
\hline $\begin{array}{l}\text { Note. CEAS = Compassionate Engagement } \\
\text { values of } p>0.05 \text { (non sig.); } d f=\text { degrees of } f \\
\text { with desired values of }<3 \text { but no }>5 \text {; SRMR }= \\
\text { values of }<.08 ; \mathrm{CFI}=\text { Comparative Fit Index, } \\
\text { = Parsimony Comparative Fit Index, with val } \\
\text { range of close to or between } .90 \text { and } .95 \text {. }\end{array}$ & $\mathrm{nd} \mathrm{Ac}$ & & $\mathrm{S}$ & & & $\begin{array}{l}\text { tatistic, } \\
\text { Chi-Sq } \\
\text { esidual, } \\
\text { tween .S } \\
\text { are with }\end{array}$ & $\begin{array}{l}\text { desired } \\
\text { statistic, } \\
\text { desired } \\
\text { d .95; PCFI } \\
\text { specified }\end{array}$ \\
\hline
\end{tabular}

